\title{
Saliency detection by selective color features
}

\author{
Yanbang Zhanga,b,*, Fen Zhang, ${ }^{\mathrm{a}, \mathrm{c}}$, Lei Guo ${ }^{\mathrm{b}}$ \\ ${ }^{a}$ College of Mathematics and Information Science, Xianyang Normal University, \\ Xianyang Shaanxi 712000, China \\ ${ }^{b}$ School of Automation, Northwestern Polytechnical University, Xi'an, Shaanxi 710072, \\ China \\ ${ }^{c}$ Department of Automatic Control, Xidian University, Xi'an, Shaanxi 710071, China
}

\begin{abstract}
This paper is concerned with selective color feature for detecting salient regions. In contrast to most existing methods related to detection in one color space, the proposed algorithm pre-segments the input image into superpixels in both RGBY color space and Lab color space. Next, to calculate color contrast we not only consider the local feature, but also compute the difference between the pixel and the whole image. In the meanwhile, based on the center-surrounding scheme, a new computational model of color distribution features is presented to detect salient regions. Finally, 2D entropy is employed as an evaluation criterion to select and integrate the proper color features. Experimental results show that the proposed method outperforms the state-of-the-art methods on salient region detection.
\end{abstract}

Keywords: visual attention, saliency model, region detection, contrast-based saliency, distribution-based saliency

\section{Introduction}

Human visual system is an intelligent processing system, which can select the important parts referred to as salient objects in a complex visual environment while ignoring others. How to detect the salient objects quickly and precisely still is a challenging and key technique. Therefore, visual

*Corresponding author at: School of Automation, Northwestern Polytechnical University, Xi'an, Shaanxi 710072, China Tel: +86 029-33720773

Email address: zhyb@mail.nwpu.edu.cn (Yanbang Zhang)

Preprint submitted to Neurocomputing

March 14, 2016 
attention mechanism has received increasing interest in recent years, partly due to various applications, such as image retrieval [1], image segmentation [2], object tracking [3], adaptive coding [4], and so on.

So far, a variety of computational models for visual saliency detection have been proposed in physiology, cognitive science, computer vision, and other fields. From the viewpoint of information processing, these algorithms can be categorized into three classes [5], namely, bottom-up model, top-down model and hybrid model. Bottom-up model simulates human instinctive visual attention mechanism, which is rapid, preattentive, and stimulus driven, and always chooses the low-level features, for example color [6], intensity [7], texture[8] to extract salient objects that are different from their surroundings. A pioneering work, called Itti model [9], put forward a saliency detection model to select the most conspicuous locations as attended regions by combining three multi-resolution local features such as luminance contrast, chrominance contrast, and orientation contrast. Motivated by this work, Judd et al. [10] introduced several new features to characterize image content which included subband pyramids based features, 3D color histogram, horizon line detector, etc.

On the other hand, since top-down model is a goal-oriented, attentive, and task-dependent manner, which uses prior knowledge to detect what we need $[11,12]$, it usually obtains more precise results than those of the bottom-up model at the sacrifice of computation. One of the most famous examples of top-down attention guidance came from Yarbus [13] in 1967, who showed that eye movements depended on the current task. Hybrid model combines the advantages of bottom-up model with top-down model, which attracts much attention recently, and many detection methods have been proposed [14] to obtain higher precision. In [14], a direct mapping was learned from bottomup and top-down features to eye fixations by resorting to SVM, AdaBoost, $k$-Nearest Neighbor algorithm(KNN), and so on.

It is worth noting that it cannot be predict salient objects before testing due to diversity and uncertainty of objects, so the existing research methods mainly focus on the bottom-up model. In this paper, we propose a bottomup model to detect objects without resorting to content or specific prior knowledge about the targets. Solving the salient object detecting problem can benefit many computer vision tasks and application. 


\section{Related Work}

When we look at an image, some objects attract our attention due to the fact that their colors are different to their surrounding areas. Since human vision is very sensitive to color, color plays an important role in detecting saliency. Achanta et al. [15] defined the color difference between every pixel and the mean value of whole image as saliency value. Achanta and Susstrunk [16] extended the work [15] to deal with each pixel in a symmetric surround rather than the whole image in the presence of local features. In the spectral domain, Hou and Zhang [17] proposed a saliency detection method by using the spectral residual of the log spectrum of an image. The model in [18] detected image saliency by analyzing the contrast in the spectral and spatial domain simultaneously, but did not involve the feature of the color distribution. Whether the pixel belongs to the salient object depends not only on pixel value of color, but also on the distribution and position of the pixel. Hence, some models that combine color contrast with color distribution to detect salient region have received more attention [19, 20, 21]. Taking advantage of color contrast and color distribution, a salient object detection model was offered in [19]. Perazzi et al. [20] proposed a contrast-based saliency model, which was consistently formulated as high-dimensional Gaussian filters. Unfortunately, they only considered the local contrast feature in Lab color space, while ignoring the information about the global ones. Based on spatialcolor constraint and similarity distribution constraint, Xu et al. [21] showed a method that measured pixel-level saliency and region-based saliency.

It is well known that feature combination is a crucial factor to improve the performance of saliency detection. In 2010, Atrey et al.[31] reviewed many state-of-the-art fusion strategies, for example, addition, multiplication, mean value, and so on. As pointed out by Zhang et al.[18], 2D entropy is a very useful tool to select the features that are combined to obtain the final saliency.

Inspired by Zhang et al.[18] and Perazzi et al.[20], we study the features of the color contrast and the color distribution in both $R G B Y$ and Lab color spaces, and propose a bottom-up model to detect the salient regions in this paper. Unlike the aforementioned methods in [20], the contrast features of local and global are considered simultaneously to detect the salient features. Moreover, we divide the combination process into two steps. First, we choose the proper channels to express the contrast and distribution features, and fuse them into the contrast feature map and the distribution feature map, respectively. Next, the final map is obtained by integrating the above results. 
Finally, comparison results show that the proposed method outperforms some existing ones.

\section{The proposed method}

In this section, we give a detailed description of the proposed saliency detection model. The section is organized as follows. The pre-segmentation model is introduced in section 3.1. The color contrast feature and the color distribution feature are considered, respectively, in sections 3.2 and 3.3. Feature combination is presented in section 3.4.

\subsection{Pre-segmentation}

Pixels that belong to the same region usually have the very similar color components, and then the computational complexity can be reduced by computing region based on contrast. Superpixel algorithms group pixels into perceptually meaningful atomic regions which have the advantages of replacement of the pixel, redundancy reduction, decreasing in complexity. In general, a good superpixel algorithm can improve the performance of the detection. Recently, Achanta [16] has proposed a new superpixel algorith$\mathrm{m}$ based on simple linear iterative clustering (SLIC), which adopts $k$-means clustering to generate superpixels, and performs an empirical comparison of the existing superpixel methods. Since human visual system is more sensible to opponent-color and Lab color space is close to the human visual attention, here, we use SLIC superpixels in RGBY color space and Lab color space, where RGBY is double opponency color space defined in [9]. In order to adapt to different salient objects, we adopts a multi-scale superpixel segmentation algorithm. For a given image, we segment it into 200, 300, 400, and 500 superpixels, respectively, and use

$$
C_{i, n}=\frac{\sum_{I_{m, n}^{C} \in R_{i}} I_{m, n}^{C}}{\left\|R_{i}\right\|}
$$

and

$$
P_{i}=\frac{\sum_{I_{m, n}^{P} \in R_{i}} I_{m, n}^{P}}{\left\|R_{i}\right\|} \quad n \in\{R B, B Y, I, L, a, b\}
$$

to denote the average color and position of superpixels in the channel $n$. where $I_{m, n}^{C}$ is the value of the pixel at the point $m$ in the channel $n .\left\|R_{i}\right\|$ is the number of pixels in superpixel region $R_{i}$. Note that, here, pre-segmenting the image into superpixels reduces redundancy and noise. 


\subsection{Contrast-based saliency}

If an object's color is different from its surrounding areas, then it is more attractive, therefore, color contrast has a significant effect on saliency detection. Commonly used color features include saturation, intensity, hue, opponents, warm and cold colors, and so on. The measurement in human visual cortex [23] has shown the strongest response is from red-green and blueyellow stimuli, which therefore absorb more human attention. In addition, Lab color space is intended to perceptual uniformity. In this space, L component closely matches human perception of lightness, and $a$ and $b$ channels approximate the human chromatic opponent system.In this paper, motivated by the fact that one color system may not always work well [7, 18], we adopt both $R G B Y$ space and Lab space, in which there exist three cases if a region is salient.

a) The color of the region is different from that of its surrounding regions and the average color of the whole image as well.

b) The color of the region is similar to the average color of the whole image, but different from that of their surrounding regions.

c) The color of the region is similar with that of its surrounding regions, but different from the average color of the whole image.

Only when the color of the region is similar with those of both their surrounding regions and the whole image, this region is the background. Fig. 1 shows some examples of three cases.

Based on the above discussion, the saliency of a superpixel in the channel $n$ is defined as

$$
\begin{gathered}
S_{S C}(i, n)=\exp \left(-\frac{\left|P_{i}-P^{c}\right|^{2}}{\sigma_{1}^{2}}\right) \sum_{\forall j \in I} W_{i, j}^{1, n}\left(D\left(C_{i, n}, C_{j, n}\right)+D\left(C_{i, n}, C_{\text {mean }, n}\right)\right) \\
n \in\{R B, B Y, I, L, a, b\}
\end{gathered}
$$

with

$$
W_{i, j}^{1, n}=\frac{1}{k} \exp \left(-\frac{\left|P_{i}-P_{j}\right|^{2}}{\sigma_{2}^{2}}\right) \exp \left(-\frac{\left|P_{j}-P^{c}\right|^{2}}{\sigma_{3}^{2}}\right)
$$

which considers the spatially weighted global contrast to all other pixels in the image and to the average color of the whole image. $D\left(C_{i, n}, C_{j, n}\right)$ denotes the Euclidean distance between the superpixels $C_{i, n}$ and $C_{j, n}, D\left(C_{i, n}, C_{\text {mean }, n}\right)$ 


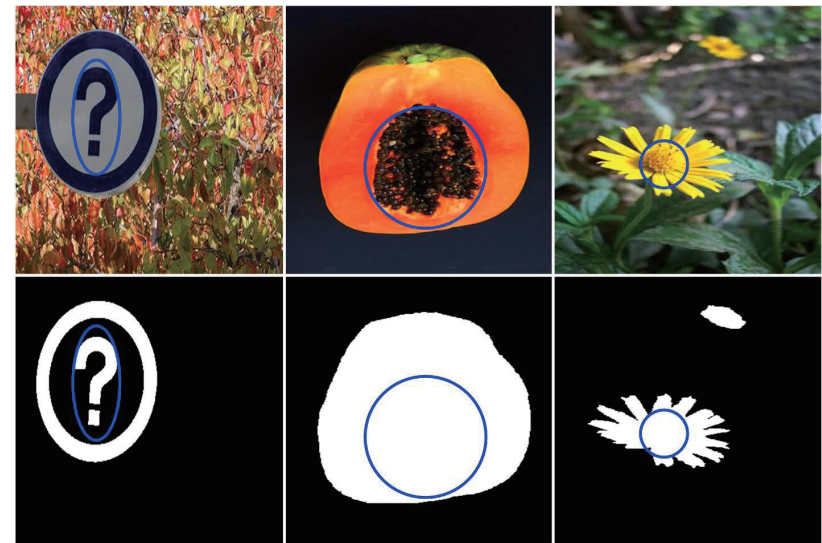

(1)

(2)

(3)

Figure 1: Three examples of saliency maps are shown. The color of the region surrounded by the blue line is different from that of its surrounding regions and the background in the sub-figure (1), and is similar to that of the background, but different from that of its surrounding regions in the sub-figure (2), and is similar to that of its surrounding regions, but different from that of the background in the sub-figure (3). The regions surrounded by the blue line in three sub-figures are parts of salient ones.

denotes the Euclidean distance between the superpixel $C_{i, n}$ and the mean pixel $C_{\text {mean }, n}$ of the whole image. $\left|P_{i}-P_{j}\right|$ means the Euclidean distance between the positions of the two superpixels. $\left|P_{i}-P^{c}\right|$ means the Euclidean distance between the position of the superpixel $P_{i}$ and the center of the image $P^{c} . \sigma_{1}$ is the control parameter for distance from the center. $\sigma_{2}$ and $\sigma_{3}$ are the control parameter for spatial similarity. From (3), we can obtain the following properties.

If both $D\left(C_{i, n}, C_{j, n}\right)$ and $D\left(C_{i, n}, C_{\text {mean }, n}\right)$ are bigger, case (a) satisfies.

If $D\left(C_{i, n}, C_{j, n}\right)$ is smaller, but $D\left(C_{i, n}, C_{\text {mean,n }}\right)$ is bigger, case (b) satisfies.

If $D\left(C_{i, n}, C_{\text {mean,n }}\right)$ is smaller, but $D\left(C_{i, n}, C_{j, n}\right)$ is bigger, case (c) satisfies. Therefore, (3) is derived to extend (1) in [20].

\subsection{Distribution-based saliency}

Since background regions generally scatter over the whole image, and the salient regions usually distribute compactly, the spatial distribution of color information is another key factor to detect the salient region. Based on the center-surrounding scheme, salient regions are usually near the center of the image and are surrounded by background regions. The spatial saliency of each superpixel in the channel $n$ is defined as 


$$
S_{S P}(i, n)=\sum_{j=1, j \neq i}^{n} \exp \left(1-d_{j}\right) W_{i, j}^{2, n} \quad n \in\{R B, B Y, I, L, a, b\}
$$

with

$$
\begin{aligned}
& d_{j}=\frac{2\left\|P_{j}-P^{c}\right\|}{\sqrt{w^{2}+h^{2}}} \\
& W_{i, j}^{2, n}=\exp \left(-\frac{\left(C_{i, n}-C_{j, n}\right)^{2}}{\delta^{2}}\right)
\end{aligned}
$$

where $w$ and $h$, respectively, denote the width and height of the image. $d_{j}$ measures the distance between the position of the pixel $P_{j}$ and the center of the image. $\delta$ is the control parameter for the difference of $C_{i, n}$ and $C_{j, n}$.

\subsection{Feature combination}

Feature combination plays an important role in improving the performance of saliency detection. As shown in [18], the saliency map is selected to represent salient features with the best performance involved, which may lead to a loss of information. On the contrary, all saliency maps are adopted, which may contain much noise and make the final saliency map get worse.

A saliency map can be regarded as a probability distribution of the pixel values. In a good salient map, the salient region should be popped out from the image, while the rest will be well suppressed. It implies that values in the saliency map would form clusters around certain values. In this case, the entropy of the saliency map should be small. Therefore, we can choose the $2 D$ information entropy as a measure of the significance of the standard, and find $N$ proper saliency maps with the smaller entropy value. As reported in [18, 22], 2D entropy is defined as: $H_{2 D}=H\left\{g_{n} * x\right\}$ where $H(x)=$ $-\sum_{i=1}^{n} p_{i} \log _{i}$, and $g_{n}$ is a low-pass Gaussian kernel.

In order to obtain the color contrast features $S^{\text {contrast }}$, we define the weight of each feature map as

$$
W_{i}=\frac{1}{K} \frac{1}{H_{2 D}\left(S_{S C}(i, n)\right)}
$$

where $K$ is a normalizing constant that guarantees $\sum_{i} W_{i}=1$, and $H_{2 D}$ is $2 \mathrm{D}$ entropy of the proper saliency maps $S_{S C}(i, n)(i=1,2, \cdots, N)$. The 
overall combination of saliency maps can be obtained by using:

$$
S^{\text {contrast }}=\sum_{i=1}^{N} W_{i} S_{S C}(i, n)
$$

Similarly, we can also obtain the color distribution features $S^{\text {distribution }}$. Next,The final map is obtained by multiplication method [24], which is expressed as

$$
\text { Smap }=S^{\text {contrast }} \cdot S^{\text {distribution }}
$$

The framework of our proposed method is shown in Fig. 2.

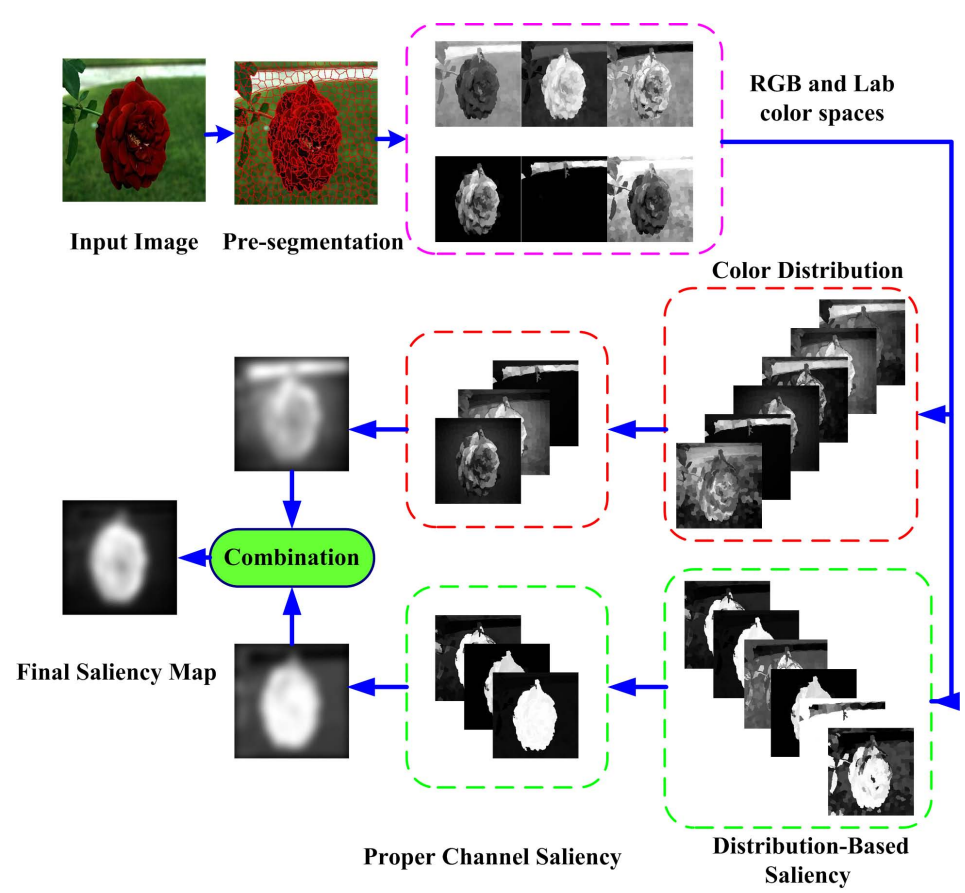

Figure 2: Architecture of the proposed saliency.

\section{Experimental results}

First, we introduce the parameters setting in the experiment. In preprocessing, the given image is segmented into meaningful atomic regions by using 
SLIC superpixels, and the number of superpixels affects the detection results. Though more superpixels can be segmented, the number of superpixels will result in a large cost of running, so the choice of $N$ is a trade-off between time-consumption and improvement of segmentation. Meanwhile, in order to adapt to different salient objects, we set the number of superpixels as 200, 300, 400, and 500, respectively. Take $\sigma_{1}^{2}=\sigma_{2}^{2}=10000, \sigma_{3}^{2}=100000$ and $\delta^{2}=64$ to computer the contrast-based saliency and the distribution-based saliency given in (3) and (5). The above parameters are relate to the size of images, and may increase with the size of images.

We test the proposed method on the Microsoft Research Asia (MSRA) dataset [15], which is publically available dataset and contains 1000 color images with ground truth using binary masks to indicate salient regions. Each image contains more than one salient object with different color, shape, size, and so on. Namely, there is no any prior knowledge about the salient objects in the images. We compare the proposed method with thirteen stateof-the-art saliency detection methods, including Itti (IT) [9], spatiotemporal cues (LC) [25], fuzzy growing (MZ) [26], graph based(GB) [10], context aware (CA) [27], spectral residual (SR) [17], frequency tuned (FT) [15], histogrambased contrast (HC) [28], region contrast (RC) [28] and hypercomplex Fourier transform (HFT) [22], Saliency filters(SF) [20], SUN [29], LIN [30]. Some comparison results from our proposed model and the others are shown in Fig. 3, from which we can see that the saliency maps from the proposed model are better than those from other existing ones.

To evaluate the performance of the proposed model, we use the popular validation approach called the receiver operating characteristics (ROC) curve [10] as the quantitative evaluation metric. The saliency maps include two different regions: salient and non-salient regions. Percentage of the salient regions from the ground-truth intersecting with the salient region from the saliency map is called true positive rate. Percentage of the non-salient regions from the ground-truth intersecting with the salient region from the saliency map is called false positive rate. Fig. 4 shows the ROC curves of various saliency detection methods calculated on all testing data. From Fig. 4, it can be seen that we can achieve performance identical to methods in [20].

For further quantitative comparisons, the area under the ROC curve (AUC) is also calculated. The larger AUC implies the performance of the saliency model is better. Table 1 shows AUCs of different algorithms. Obviously, the proposed method achieves a better performance in terms of ROC curve and AUC score, which demonstrates the superiority of our work. 


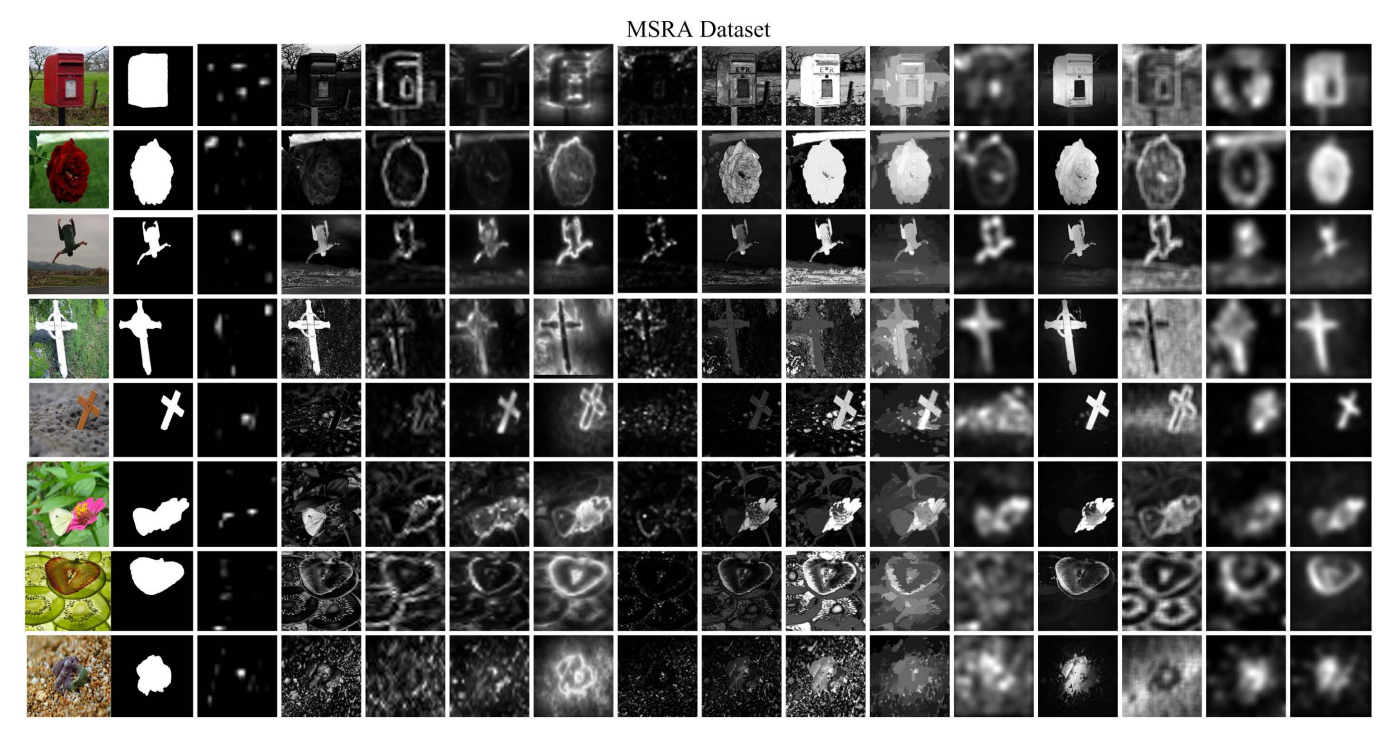

Figure 3: Examples of saliency maps on the MSRA dataset. From left to right, there are original images, ground truth masks, and saliency maps achieved by the methods IT [9], LC [25], MZ [26], GB [10], CA [27], SR [17], FT [15], HC [28], RC [28], HFT [22], SF[20], SUN[29] ,LIN[30] and the proposed method, respectively.
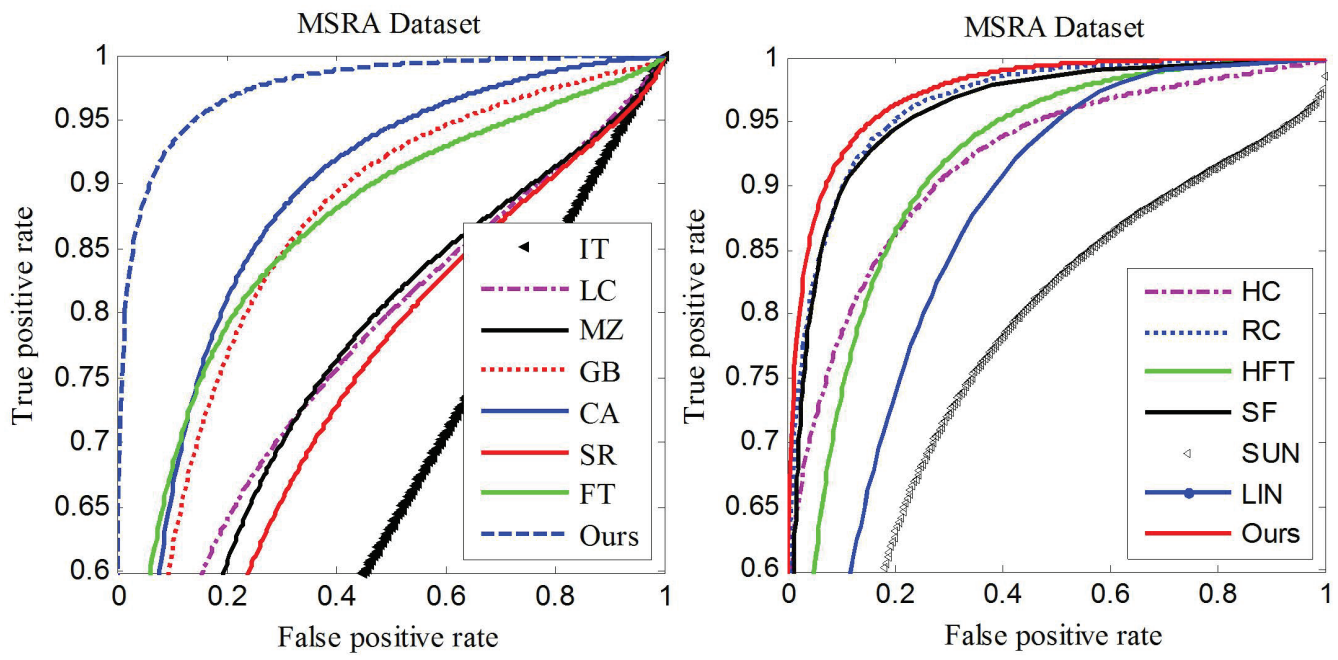

Figure 4: Comparison of different algorithms by ROC. 
Table 1: Comparison of Different Algorithms by AUC

\begin{tabular}{cccccccc}
\hline Method & IT & LC & MZ & GB & CA & SR & FT \\
\hline AUC & 0.6295 & 0.7687 & 0.7481 & 0.8550 & 0.8779 & 0.7234 & 0.8642 \\
\hline Method & HC & RC & HFT & SF & SUN & LIN & Ours \\
AUC & 0.9181 & 0.9639 & 0.9118 & 0.9596 & 0.7590 & 0.8448 & 0.9728 \\
\hline
\end{tabular}

To evaluate the effect of color channels to saliency detection, we perform an experiment with $N$ proper saliency maps. Results on the MSRA database are shown in Table 2. Our results show that AUC reaches a maximum when $N=4$, while the method [18] reaches the maximum at $N=3$. At $N=1,2$, the results of the proposed method is worse than the model [18], however, the results at $N=3,4,5,6$ outperform the all existing works.

Table 2: Comparison with Method [18] at Different Channels by AUC

\begin{tabular}{ccccccc}
\hline$N$ & 1 & 2 & 3 & 4 & 5 & 6 \\
\hline Ours & 0.9447 & 0.9669 & 0.9714 & 0.9728 & 0.9723 & 0.9693 \\
method [18] & 0.9517 & 0.9634 & 0.9672 & 0.9669 & 0.9656 & 0.9617 \\
\hline
\end{tabular}

To evaluate the effect of color spaces on saliency detection, we perform an experiment by using each of the two color systems with $N$ proper saliency maps. The results of $A U C$ in $L a b, R G B Y$ and combination of them are listed in Table 3, respectively, which indicates that the integration of both color systems can lead to higher accuracy compared with using either of them.

Table 3: Comparison of AUC in color spaces with different $N$

\begin{tabular}{ccccccc}
\hline$N$ & 1 & 2 & 3 & 4 & 5 & 6 \\
\hline LAB & 0.9357 & 0.9576 & 0.9613 & & & \\
RGBY & 0.9555 & 0.9682 & 0.9699 & & & \\
LAB+RGBY & 0.9447 & 0.9669 & 0.9714 & 0.9728 & 0.9723 & 0.9693 \\
\hline
\end{tabular}

To fully evaluate the performance of the proposed model, we adopt the commonly used precision and recall measures, and plot the precision-recall 
curves to compare it with aforementioned thirteen state-of-the-art saliency detection methods. Each saliency map is normalized into the same range of $[0,255]$, and then we vary the integer threshold from 0 to 255 to obtain 256 binary salient object masks. Let $S$ and $G$ denoting each binary salient object mask and the corresponding ground truth, respectively, the precision and recall can are defined as

$$
\begin{aligned}
\text { precision } & =\frac{\sum_{(x, y)} S(x, y) \cdot G(x, y)}{\sum_{(x, y)} S(x, y)} \\
\text { recall } & =\frac{\sum_{(x, y)} S(x, y) \cdot G(x, y)}{\sum_{(x, y)} G(x, y)}
\end{aligned}
$$
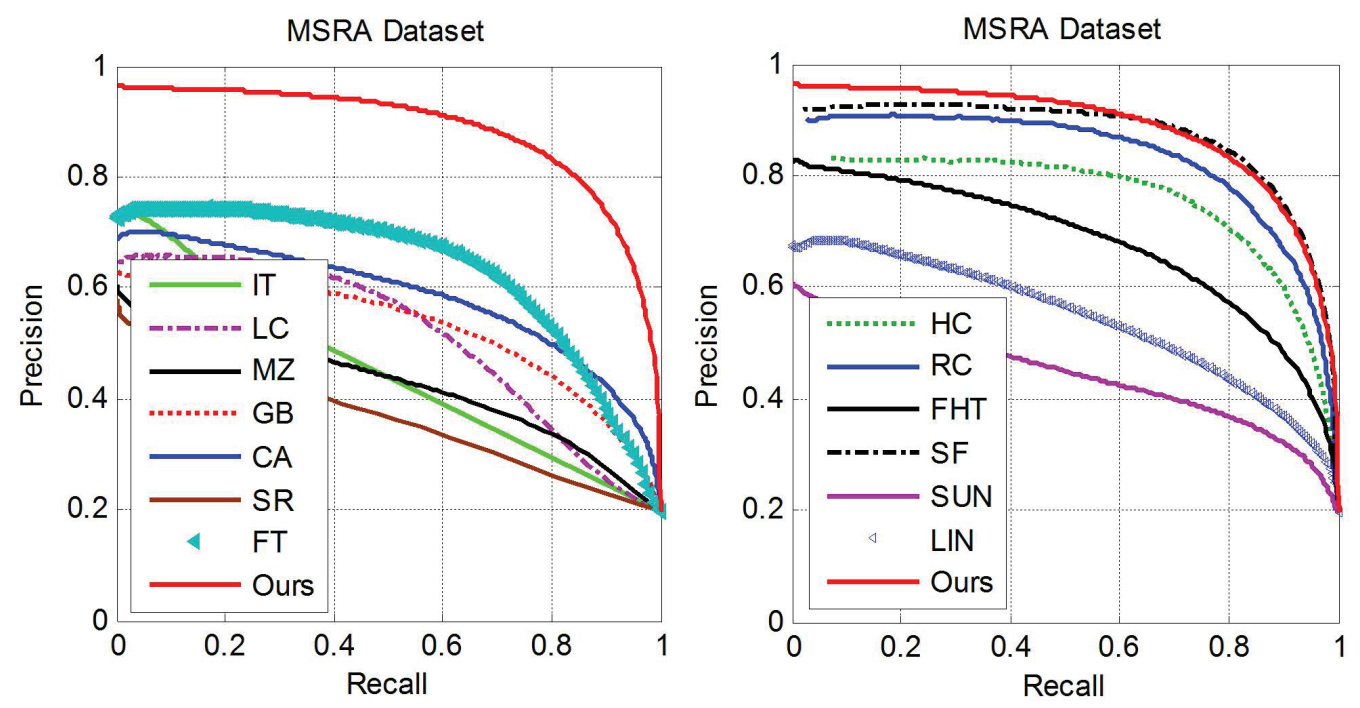

Figure 5: Precision-recall curves of different saliency models.

For each class of saliency maps, at each threshold, the precision and recall measures calculated for all 1000 images are averaged, and the corresponding precision-recall curve plots the 256 average precision values versus the 256 average recall values. The curves of precision versus recall for different saliency maps are shown in Fig. 5. It can be seen that the proposed method shows the highest precision for most of the recall values except the SF method. Compared with SF method, the proposed method has a similar precision of the recall values. From the right sub-figure of Fig. 5, we obtain the best result 
than above-mentioned methods when the value of the recall is less than 0.6, while we have the similar performance with the SF method when the value is greater than 0.6. It means that the saliency maps obtained by the proposed method have the better performance in highlighting salient regions in the MSRA dataset.

Inspired by [18] and [20], and we fully analysis the color features, and point out when the pixel belongs to the salient region. The contrast features in both local and global are considered simultaneously, and $2 D$ entropy is used as an evaluation criterion to select and integrate the proper color features. So the proposed method outperforms the state-of-the-art methods in ROC curves and AUC. While we have only considered the color feature in this paper, there may exist omissions when the color of an object is similar to that of background. Therefore, in the comparison of precision-recall curves, we obtain the similar performance with SF method when the value of recall is greater than 0.6.

\section{Conclusion}

In this paper, we present a novel saliency detection model by using the color contrast features and color distribution features. Based on the global contrast features and local contrast features, omissions are greatly reduced. Moreover, 2D entropy as an evaluation criterion is used to select the proper subsets of saliency maps that are fused together to obtain the final map, which can highly reduce false detection. Finally, experimental results are provided to show that the proposed method outperforms the state-of-the-art methods on salient region detection.

A major limitation of the proposed work is that it only adopts the color information. However, other factors, such as texture and shape information, may also be useful for saliency detection. Future work will take into account more bottom-up factors that can further improve the performance and extend this model to saliency detection in dynamic videos.

\section{Acknowledgements}

This research was supported by the National Science Foundation of China (61273362, 61501388, 61401357), the Specialized Research Fund of Xianyang Normal University (13XSYK009, 14XSYK005) 
[1] Y. Tsai, Hierarchical salient point selection for image retrieval, Pattern Recognition Letters, 33(12) (2012) 1587-1593.

[2] X. Bai, W. Wang, Saliency-SVM: An automatic approach for image segmentation. Neurocomputing, 136 (2014) 243-255.

[3] M. Guo, Y. Zhao, C. Zhang, et al. Fast object detection based on selective visual attention. Neurocomputing, 2014, 144: 184-197.

[4] Z. Chen, J. Han, K. Ngan, Dynamic bit allocation for multiple video object coding, IEEE Transactions on Multimedia. 8 (2006) 1117-1124.

[5] L. Zhang, W. Lin, Selective Visual Attention: Computational Models and Applications, John Wiley and Sons, 2013.

[6] Y. Zhang, J. Han, L. Guo. Salient region detection using background priors. Optik-International Journal for Light and Electron Optics, 125(19) (2014) 5872-5877.

[7] A. Borji, Boosting bottom-up and top-down visual features for saliency estimation, in: IEEE Conference on Computer Vision and Pattern Recognition, 2012, pp. 438-445.

[8] H. Chen, J. Leou, A new visual attention model using texture and object features, in: IEEE Conference on Computer and Information Technology Workshops, 2008, pp. 374-378.

[9] L. Itti, C. Koch, E. Niebur, A model of saliencybased visual attention for rapid scene analysis, IEEE Transactions on Pattern Analysis and Machine Intelligence, 20 (1998) 1254-1259.

[10] J. Harel, C. Koch, and P. Perona, Graph-based visual saliency, Advances in Neural Information Processing Systems, 19 (2007) 545 -552.

[11] D. Gao, S. Han, N. Vasconcelos, Discriminant saliency, the detection of suspicious coincidences, and applications to visual recognition, IEEE Transactions on Pattern Analysis and Machine Intelligence, 31(6) (2009) 989-1005.

[12] P. Baldi, L. Itti, Of bits and wows: a Bayesian theory of surprise with applications to attention, Neural Networks, 23(5) (2010) 649-666. 
[13] A. L. Yarbus, Eye-Movements and Vision, Plenum Press, New York, 1967.

[14] B. Wu L. Xu, Integrating bottom-up and top-down visual stimulus for saliency detection in news video, Multimedia Tools and Applications, 73(3) (2013) 1-23.

[15] R. Achanta, S. Hemami, F. Estrada, S. Susstrunk, Frequency-tuned salient region detection, in: IEEE Conference on Computer Vision and Pattern Recognition, 2009, pp. 1597-1604.

[16] R. Achanta, A. Shaji, K. Smith, A. Lucchi, P. Fua, S. Susstrunk, SLIC superpixels compared to state-of-the-art superpixel methods, IEEE Transactions on Pattern Analysis and Machine Intelligence, 34(11) (2012) 2274 - 2281.

[17] X. Hou, L. Zhang, Saliency detection: A spectral residual approach, in: IEEE Conference on Computer Vision and Pattern Recognition, 2007, pp. 1-8.

[18] Y. Zhang, J. Han, L. Guo, Saliency detection by combining spatial and spectral information, Optics Letters, 38(11) (2013) 1987-1989.

[19] K. Fu, C. Gong,J. Yang, Y. Zhou, Salient object detection via color contrast and color distribution, in: Asian Conference on Computer Vision, 2012, pp. 111-122 .

[20] F. Perazzi, P. Krahenbuhl, Y. Pritch, A. Hornung, Saliency filters: contrast based filtering for salient region detection, in: IEEE Conference on Computer Vision and Pattern Recognition, 2012, pp. 733-740.

[21] L. Xu, H. Li, L. Zeng, K. Ngan, Saliency detection using joint spatialcolor constraint and multi-scale segmentation, Journal of Visual Communication and Image Representation, 24(4) (2013) 465-476.

[22] J. Li, M. Levine, X. An, X. Xu, and H. He, Visual saliency based on scale-space analysis in the frequency domain, IEEE Transactions on Pattern Analysis Machine Intelligence, 35(4) (2013) 996-1010.

[23] S. Engel, X. Zhang, Wandell B. Colour tuning in human visual cortex measured with functional magnetic resonance imaging. Nature, 388(6637) (1997) 68-71. 
[24] I. Gonzlez-Daz, H. Boujut, V. Buso, J. Benois-Pineau, D. Jean-Philippe, Saliency-based object recognition in video, http://hal.archivesouvertes.fr/hal-00799127 (2013).

[25] Y. Zhai, and M. Shah, Visual attention detection in video sequences using spatiotemporal cues. Proceedings of the 14th annual ACM international conference on Multimedia, (2006) 815-824.

[26] Y. Ma and H. Zhang, Contrast-based image attention analysis by using fuzzy growing, in: ACM international conference on Multimedia, 2008, pp. 374-381.

[27] S. Goferman, L. Zelnik-Manor, and A. Tal, Context-aware saliency detection, IEEE Transactions on Pattern Analysis and Machine Intelligence, 34(10) (2012) 1915-1926.

[28] M. Cheng, G. Zhang, N. Mitra, X. Huang, and S. Hu, Global contrast based salient region detection, in: IEEE Conference on Computer Vision and Pattern Recognition, 2011, pp. 409-416.

[29] L. Zhang, M. H. Tong, T. K. Marks, H. Shan, and G. W. Cottrell, SUN: A Bayesian Framework for Saliency Using Natural Statistics, Journal of Vision, 8(7) (2008) 1-20.

[30] Y. Lin, Y. Y. Tang, B. Fang, et al, A visual-attention model using earth mover's distance-based saliency measurement and nonlinear feature combination, IEEE Transactions on Pattern Analysis and Machine Intelligence, 35(2) (2013) 314-328.

[31] P. K. Atrey, et al.,Multimodal fusion for multimedia analysis: a survey, Multimedia systems 16(6) (2010) 345-379. 